\title{
Volatility Spillovers Between Oil Prices And Stock Returns: A Focus On Frontier Markets
}

Mathieu Gomes, Université d'Auvergne Clermont, France Anissa Chaibi, IPAG Business School, France

\begin{abstract}
Frontier markets are increasingly sought by investors in search of higher returns and low correlation with traditional assets. As such, it is important for financial market participants to understand the volatility transmission mechanism across these markets in order to make better portfolio allocation decisions. This paper employs a bivariate BEKK-GARCH $(1,1)$ model to simultaneously estimate the mean and conditional variance between equity stock markets (twentyone national frontier stock indices and two broad indices - the MSCI Frontier Markets and the MSCI World) and oil prices. We examine weekly returns from February 8, 2008 to February 1, 2013 and find significant transmission of shocks and volatility between oil prices and some of the examined markets. Moreover, this spillover effect is sometimes bidirectional.
\end{abstract}

Keywords: Volatility Spillovers; Oil Prices; Stock Returns; Multivariate GARCH; Diversification; Frontier Markets

\section{INTRODUCTION}

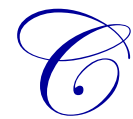

rude oil is probably the physical commodity that most affects the state of the economy. This impact can take place through various paths. When crude oil prices rise, cost of production of goods and services increases, and so do transportation and heating costs. This, in turn, can lead to inflationary pressures and thus restricted consumption from consumers. This produces a negative effect to capital markets, consumer confidence, and the macroeconomy as a whole. There are thus, various theoretical reasons for which oil prices can have a significant impact on financial markets, and stock markets in particular. One of them is that the value of stock prices in equity asset valuation models theoretically equals the sum of future discounted earnings expectation of companies or future cash flows. Therefore, changes in crude oil prices can directly have an impact on stock prices through the expected cash flows on the one hand, and through the discount rate on the other hand.

The first and most intuitive way through which oil prices can affect stock prices is by the channel of expected cash flows. Oil is a crucial input in the production of goods and services, and therefore, a rise in oil prices is likely to increase production costs, which in turn will reduce margins, cash flows and thus stock prices. But oil prices can also affect stock valuations through the rate that is used to discount future cash flows. Indeed, rising oil prices can create inflationary pressures that will ultimately lead to the decision-making by central banks to tighten their monetary policies and raise interest rates. This can have tremendous implications for companies as corporate investment decisions can be affected directly by changes in the discount rate. Moreover, a change in interest rates will impact company financing through higher borrowing costs and a lower market value relative to book value, which will further damage a company's ability to raise new funds.

However, it is worth noting that not all companies will react the same way to changes in crude oil prices. Indeed, the direction of stock price reactions will depend on whether the company is an oil producer or an oil consumer. Oil producers will profit from an oil price increase while oil consumers will suffer from it. Overall, since the great majority of companies are oil consumers, it is logical to expect a negative effect of oil prices on stock prices. 
To the better of our knowledge, no previous study has examined the effect of oil price shocks on frontier markets. Our paper aims to fill this gap by making use of a rich database and innovative econometric techniques. Section 2 discusses findings of previous works on interactions between oil prices and stock markets with a specific focus on emerging markets. Section 3 introduces the concept of frontier markets. A preliminary analysis of our database is provided in Section 4. Section 5 introduces our empirical methodology. Results are discussed in Section 6 , while Section 7 concludes and gives some policy implications.

\section{LITERATURE REVIEW}

A number of research papers have explicitly examined the relationship between oil markets and economic variables, such as GDP growth rates, inflation, employment, and exchange rates (Hamilton, 1983; Gisser \& Goodwin, 1986; Mork, 1989; Hooker, 1996; among others). The impact of oil price changes on the world economy is indeed large. According to Adelman (1993), "Oil is so significant in the international economy that forecasts of economic growth are routinely qualified with the caveat: 'Provided there is no oil shock." "As another proof of that importance, the International Monetary Fund (2000) estimated that a US\$5 per barrel price increase had tremendous effects on the state of the economy with a reduction of global economic growth by $0.3 \%$ in the following year.

Surprisingly, while understanding the relationship between oil price changes and stock markets may appear crucial to energy policy planning, energy risk management and portfolio diversification, these links have only been examined recently. Jones and Kaul (1996) were the first to test the reaction of international stock markets (Canada, UK, Japan, and USA) to oil price shocks, based on the standard cash-flow dividend valuation model. They find that for Canada and the US, this reaction can be entirely accounted for by the impact of the oil shocks on cash flows. Huang et al. (1996), using an unrestricted Vector Autoregressive (VAR) model, show a significant link between the stock returns of certain American oil companies and oil price changes. However, there is no evidence of a link between oil prices and market indices such as the S\&P 500. In contrast, Sadorsky (1999), using a Vector Autoregressive (VAR) framework, shows that oil prices play an important role in affecting economic activity. His results also suggest an asymmetric relationship, as changes in economic activity don't seem to have an impact on oil prices.

Ciner (2001), using non-linear causality tests, provides empirical evidence that oil shocks significantly affect stock index returns in the US in a non-linear manner, and that the returns also have impacts on crude oil futures. Park and Ratti (2008) show that oil price shocks have a statistically significant impact on real stock returns contemporaneously and/or within the following month in the U.S. and 13 European countries over the period running from January 1986 to December 2005 and that Norway, as an oil exporter, shows a statistically significantly positive response of real stock returns to an oil price increase.

More recently, some studies have examined the extent of oil price impacts on stock prices from a sector-bysector perspective. For example, El-Sharif et al. (2005) show that the stock returns of UK Oil \& Gas companies are positively correlated to oil price increases. Boyer and Filion (2007) obtain similar results for Oil \& Gas returns in Canada. Arouri and Nguyen (2010), using various econometric techniques, suggest that the sensitivity of European sector stock returns to oil price changes greatly differ from one sector to another, with Oil \& Gas stocks profiting from oil price increases. Similarly, Arouri, Bellalah, and Nguyen (2011) show that, on the basis of short-term analysis, strong positive links are found in some GCC (Gulf Cooperation Council) countries between oil prices and stock markets, and that this causality generally runs from oil prices to stock markets.

Despite various studies focusing on price spillovers between oil and stock markets, it is only recently that some attention has been paid to possible volatility spillovers between these two markets. Using a multivariate GARCH model, Malik and Hammoudeh (2005) find significant volatility transmission between second moments of the US equity and global oil markets. In that same study, they find that the three examined Gulf equity markets (Bahrain, Kuwait, and Saudi Arabia) receive volatility from the oil market, with Saudi Arabia featuring an interesting characteristic, i.e. a significant volatility spillover from the Saudi equity market to the global oil market, underlining the major role played by Saudi Arabia in the global oil market. Agren (2006), using an asymmetric BEKK model, finds strong evidence of volatility spillovers (albeit relatively small) from oil prices to stock markets in Japan, Norway, the UK, and the US. Malik and Ewing (2009) study volatility spillovers between oil prices and 
five US equity sector indices (Financials, Industrials, Consumer Services, Health Care, and Technology) and conclude in favor of significant transmission of shocks and volatility between oil prices and some of the examined market sectors.

Using a recent generalized VAR-GARCH approach to examine the extent of volatility transmission between oil and stock markets in Europe and the US at the sector level, Arouri, Jouini, and Nguyen (2011) find evidence of significant volatility spillover. Their study suggests that the transmission is usually unidirectional from oil markets to stock markets in Europe, but bidirectional in the US. Chang et al. (2012), using various econometric models, investigate the conditional correlations and volatility spillovers between the crude oil and financial markets, and find little evidence of volatility transmission between the oil market and major stock indices (FTSE100, Dow Jones, and S\&P500). These results would tend to confirm that volatility transmission between oil and stock markets only occurs in some sectors.

\section{A FOCUS ON "FRONTIER MARKETS"}

To the best of my knowledge, no research has so far been done about possible volatility transmission between oil markets and frontier markets (apart from GCC countries). The term "frontier markets" was coined by the International Finance Corporation (IFC), a private sector arm of the World Bank Group, in 1992 to reflect a subset of emerging market economies. Frontier markets are investable but have lower market capitalization (usually under 17\% of GDP) and liquidity than the more developed "traditional" emerging markets, which makes them inherently riskier investments but also provides potential opportunities for investors to take advantage of privatizations and increased listings on local exchanges over time. As a result, they may also provide better returns. Investments in these markets are thus generally pursued by investors who are seeking higher returns, and who are willing to assume the higher level of risk associated with such markets. Among these risks are political instability, poor liquidity, inadequate regulation, substandard financial reporting, and large currency fluctuations. Apart from risks, investing in frontier markets is a smart diversification to an equity portfolio, as these markets have comparatively less correlation to developed markets, and in good times, can likely beat the returns of other markets.

Despite the growing attention to frontier markets among the investment community, very little research actually includes them. However, the contributions to this particular topic reach similar conclusions: frontier markets may offer promising diversification benefits due to low correlations with developed equity markets (Speidell \& Krohne, 2007; Jayasuriya \& Shambora 2009; Berger, Pukthuanthong, \& Yang, 2011).

No official list of frontier markets countries has so far been established, but the number usually ranges between twenty-five and thirty countries. Oil economies often have the largest representation in frontier market indices. For example, five Gulf Cooperation Council (GCC) countries (Qatar, UAE, Oman, Bahrain, and Kuwait) currently make up about $60 \%$ of the MSCI Frontier Markets Index. Frontier markets are widely diverse in terms of income, geography and degree of economic development. For example, the GCC countries are among the richest economies globally on a per capita basis, while many of the key Sub-Saharan economies are among the poorest (the average GDP per capita of GCC economies is approximately $\$ 43,300$ PPP while the average GDP per capita of the frontier Africa and Asia countries is under \$2,000 PPP). But the middle class is growing in many frontier markets as improvements in logistics, better access to technology, the spread of mobile phones and better use of natural resources have begun to raise millions out of poverty while boosting consumption trends.

\section{DATA SET}

Equity market data are obtained from MSCI. In this paper, we use a set of data consisting of twenty-one national aggregate stock market indices (Argentina, Bahrain, Bulgaria, Jordan, Kazakhstan, Kenya, Kuwait, Lebanon, Mauritius, Nigeria, Oman, Pakistan, Qatar, Romania, Saudi Arabia, Slovenia, Sri Lanka, Tunisia, United Arab Emirates, Ukraine, and Viet-Nam) and two broader stock indices (MSCI Frontier Markets and MSCI World), together with a measure of the world oil price (Brent). All data are at the weekly frequency and cover a five-year period ranging from February 8, 2008 to February 1, 2013, yielding a total of 261 observations. We use weekly data because as suggested by Arouri and Nguyen (2010), they appear to be less noisy than daily data, while still capturing much of the information content of stock indices and oil prices. 
Table 1: Summary Statistics for Weekly Percentage Returns on Twenty-One National Stock Market Indices, Two Broad Stock Indices and the Oil Price

\begin{tabular}{|c|c|c|c|c|c|c|c|}
\hline & \multicolumn{2}{|c|}{ ARG } & ВАН & BRENT & \multicolumn{2}{|c|}{ BUL } & FM \\
\hline Mean & \multicolumn{2}{|c|}{-0.000392} & -0.006716 & 0.002214 & \multicolumn{2}{|c|}{-0.005666} & -0.002289 \\
\hline Median & \multicolumn{2}{|c|}{0.002725} & -0.003219 & 0.005534 & \multicolumn{2}{|c|}{-0.002640} & 0.000000 \\
\hline Maximum & \multicolumn{2}{|c|}{0.190511} & 0.083382 & 0.273051 & \multicolumn{2}{|c|}{0.193988} & 0.072381 \\
\hline Minimum & \multicolumn{2}{|c|}{-0.264352} & -0.191904 & -0.223806 & \multicolumn{2}{|c|}{-0.340314} & -0.141460 \\
\hline Std. Dev. & \multicolumn{2}{|c|}{0.061570} & 0.031999 & 0.051031 & \multicolumn{2}{|c|}{0.050725} & 0.025259 \\
\hline Skewness & \multicolumn{2}{|c|}{-0.648030} & -1.713767 & -0.238456 & \multicolumn{2}{|c|}{-1.161328} & -1.694278 \\
\hline Kurtosis & \multicolumn{2}{|c|}{6.587412} & 11.23679 & 7.276525 & \multicolumn{2}{|c|}{11.67068} & 10.88979 \\
\hline Jarque-Bera & \multicolumn{2}{|c|}{158.2236} & 865.5709 & 201.3627 & \multicolumn{2}{|c|}{876.2579} & 801.8263 \\
\hline Probability & \multicolumn{2}{|c|}{0.000000} & 0.000000 & 0.000000 & \multicolumn{2}{|c|}{0.000000} & 0.000000 \\
\hline Observations & \multicolumn{2}{|c|}{261} & 261 & 261 & \multicolumn{2}{|c|}{261} & 261 \\
\hline & JOR & KAZ & KEN & KUW & LEB & MAU & NIG \\
\hline Mean & -0.002876 & -0.000785 & 0.001149 & -0.002344 & -0.000651 & -0.000475 & -0.001280 \\
\hline Median & -0.001944 & -0.000461 & 0.002064 & -0.000693 & -0.002999 & -0.002000 & -0.000537 \\
\hline Maximum & 0.096996 & 0.339806 & 0.237935 & 0.182310 & 0.160940 & 0.117474 & 0.199916 \\
\hline Minimum & -0.129781 & -0.275896 & -0.144212 & -0.192224 & -0.109269 & -0.200252 & -0.180769 \\
\hline Std. Dev. & 0.028759 & 0.055016 & 0.037551 & 0.037484 & 0.030326 & 0.034643 & 0.047633 \\
\hline Skewness & -0.588160 & 0.345285 & 0.660617 & -0.519418 & 1.157897 & -0.561908 & -0.006575 \\
\hline Kurtosis & 6.526772 & 10.74318 & 9.986814 & 9.539805 & 10.11749 & 8.666132 & 6.297023 \\
\hline Jarque-Bera & 150.3126 & 657.2164 & 549.8534 & 476.8495 & 609.2340 & 362.8772 & 118.2170 \\
\hline Probability & 0.000000 & 0.000000 & 0.000000 & 0.000000 & 0.000000 & 0.000000 & 0.000000 \\
\hline \multirow[t]{2}{*}{ Observations } & 261 & 261 & 261 & 261 & 261 & 261 & 261 \\
\hline & OMA & PAK & QAT & ROM & SAU & SLO & SRI \\
\hline Mean & -0.001893 & -0.001231 & 0.000503 & -0.000461 & -0.000288 & -0.003351 & 0.002483 \\
\hline Median & 0.000857 & 0.002903 & 0.000849 & 0.007082 & 0.001653 & -0.000979 & -0.001682 \\
\hline Maximum & 0.118208 & 0.166830 & 0.137242 & 0.222458 & 0.144495 & 0.120431 & 0.421636 \\
\hline Minimum & -0.199966 & -0.238645 & -0.203556 & -0.293018 & -0.166560 & -0.197436 & -0.131955 \\
\hline Std. Dev. & 0.033284 & 0.044879 & 0.037994 & 0.058200 & 0.038431 & 0.037662 & 0.045690 \\
\hline Skewness & -1.395272 & -1.124315 & -0.930632 & -0.819155 & -0.376414 & -0.979714 & 3.529371 \\
\hline Kurtosis & 11.32211 & 9.013421 & 9.588668 & 6.868070 & 6.752524 & 7.744587 & 31.31229 \\
\hline Jarque-Bera & 837.8604 & 448.2410 & 509.7639 & 191.9005 & 159.2990 & 286.5613 & 9259.100 \\
\hline Probability & 0.000000 & 0.000000 & 0.000000 & 0.000000 & 0.000000 & 0.000000 & 0.000000 \\
\hline Observations & 261 & 261 & 261 & 261 & 261 & 261 & 261 \\
\hline & TU & & UAE & UKR & & & WORLD \\
\hline Mean & 0.00 & & -0.001789 & -0.006956 & & 1192 & 0.000362 \\
\hline Median & 0.00 & & -0.001154 & -0.007190 & & 0214 & 0.002840 \\
\hline Maximum & 0.09 & & 0.230320 & 0.251340 & & 965 & 0.123224 \\
\hline Minimum & -0.12 & & -0.278740 & -0.204843 & & 5901 & -0.200515 \\
\hline Std. Dev. & 0.02 & & 0.050793 & 0.056337 & & 716 & 0.032802 \\
\hline Skewness & -0.52 & & -1.007268 & 0.197675 & & 316 & -0.915448 \\
\hline Kurtosis & 7.22 & & 9.837133 & 5.131759 & & 099 & 8.946894 \\
\hline Jarque-Bera & 205. & & 552.5015 & 51.12010 & & 734 & 421.0553 \\
\hline Probability & 0.00 & & 0.000000 & 0.000000 & & 125 & 0.000000 \\
\hline Observations & 26 & & 261 & 261 & & & 261 \\
\hline
\end{tabular}

We can see from Table 1 that out of twenty-one national aggregate equity indices, seventeen display negative average weekly returns, which is not surprising given the characteristics of the covered period and the impact of the economic and financial crisis since 2008. The broader MSCI Frontier Markets index displays a negative average weekly return of $-0.2289 \%$ while the MSCI World index exhibits a positive average weekly return of $0.0362 \%$, largely driven by a recent bull market in the United States which represent the largest constituent of the index. The Brent posted an average weekly return of $0.2214 \%$ over the period.

At the country level, the highest weekly mean return is posted by the Sri Lanka stock market with a weekly average of $0.25 \%$ while the lowest average weekly return comes from Ukraine $(-0.70 \%)$. The highest weekly return was posted by Sri Lanka on the week ranging from May 18, 2009 to May 22, 2009 (+42\%), followed by Kazakhstan (+34\% on the week ranging from October 27, 2008 to October 31, 2008). 
The lowest return comes from Bulgaria $(-34 \%)$ on the week ranging from October 6, 2008 to October 10, 2008, followed by neighboring Romania (-29\% on the week ranging from January 5, 2009 to January 9, 2009). The Brent gained up to $27 \%$ (from December 29, 2008 to January 2, 2009) while its biggest weekly drop was $-22 \%$ (from December 1, 2008 to December 5, 2008). Over the whole period, the best performer was Sri Lanka with a $49 \%$ gain while the worst performer was the Ukrainian stock market, which lost approximately $90 \%$ of its value.

Weekly standard deviations range from $2.67 \%$ (Tunisia) to 6.16\% (Argentina). The MSCI Frontier Markets index varied $2.53 \%$ while the MSCI World index and the Brent experienced volatilities of $3.28 \%$ and $5.10 \%$ respectively.

All return series display non-zero skewness and excess kurtosis. This feature associated with significant Jarque-Bera statistics, indicate that the returns are not normally distributed. Prior to estimating the conditional mean or conditional variance, it is sensible to test for unit roots in the series. Standard unit root testing procedures based on the Augmented Dickey-Fuller (ADF) and Phillips-Perron (PP) tests are used. Table 2 features the results for levels (price series in logarithms) and first differences (return series) of oil and stock market price indices.

The results from the Augmented Dickey-Fuller (ADF) tests indicate that the price series are integrated of order one, except for the MSCI Frontier Markets Index, Pakistan, Slovenia (all of them at the $10 \%$ level) and Viet Nam (1\% level) stock market prices, which are stationary. The Phillips Perron test reaches similar conclusions for Pakistan (10\% level) and Viet Nam (1\% level). Alternatively, all the return series do not have unit roots according to both tests (all at the $1 \%$ level).

Table 2: Unit Root Tests

\begin{tabular}{|c|c|c|c|c|}
\hline & ADF test in Level & ADF test in Returns & PP test in Level & PP test in Returns \\
\hline ARG & -1.2742 & $-9.9597 * * *$ & -1.4877 & $-17.7236 * * *$ \\
\hline ВАН & -2.1382 & $-6.6618 * * *$ & -1.8080 & $-15.2110 * * *$ \\
\hline BRENT & -1.2237 & $-16.4203 * * *$ & -1.4678 & $-16.4797 * * *$ \\
\hline BUL & -2.4516 & $-5.8800 * * *$ & -2.1701 & $-14.9115 * * *$ \\
\hline FM & $-2.7704 *$ & $-5.8306 * * *$ & -2.4363 & $-14.7141 * * *$ \\
\hline JOR & -1.8068 & $-18.1447 * * *$ & -1.8263 & $-18.0874 * * *$ \\
\hline KAZ & -2.2125 & $-14.8040 * * *$ & -2.3390 & $-14.8001 * * *$ \\
\hline KEN & -1.5248 & $-13.2526 * * *$ & -1.3717 & $-13.4432 * * *$ \\
\hline KUW & -2.4161 & $-15.961 * * *$ & -2.4157 & $-15.9613 * * *$ \\
\hline LEB & -0.9143 & $-14.9522 * * *$ & -1.4812 & $-15.8864 * * *$ \\
\hline MAU & -2.0457 & $-13.8381 * * *$ & -2.0416 & $-13.9397 * * *$ \\
\hline NIG & -2.3870 & $-14.7988 * * *$ & -2.3704 & $-14.8091 * * *$ \\
\hline OMA & -1.7742 & $-17.7192 * * *$ & -1.8822 & $-17.8132 * * *$ \\
\hline PAK & $-2.6197^{*}$ & $-13.9072 * * *$ & $-2.6308^{*}$ & $-14.1896 * * *$ \\
\hline QAT & -1.6244 & $-15.6487 * * *$ & -1.7169 & $-15.6487 * * *$ \\
\hline ROM & -2.2862 & $-8.7771 * * *$ & -2.2802 & $-14.6471 * * *$ \\
\hline SAU & -2.2619 & $-17.9140 * * *$ & -2.2120 & $-17.9029 * * *$ \\
\hline SLO & $-2.5811 *$ & $-17.0239 * * *$ & -2.5143 & $-17.1002 * * *$ \\
\hline SRI & -0.8899 & $-13.9244 * * *$ & -1.0216 & $-14.330 * * *$ \\
\hline TUN & -2.3751 & $-17.0209 * * *$ & -2.3728 & $-17.0144 * * *$ \\
\hline UAE & -2.4923 & $-15.7630 * * *$ & -2.4924 & $-15.8874 * * *$ \\
\hline UKR & -2.1153 & $-7.8223 * * *$ & -1.9369 & $-12.6360 * * *$ \\
\hline VIE & $-3.7097 * * *$ & $-13.9400 * * *$ & $-3.8116 * * *$ & $-13.9291 * * *$ \\
\hline WORLD & -1.8818 & $-16.9366 * * *$ & -1.8818 & $-16.9174 * * *$ \\
\hline
\end{tabular}

Note: ADF and PP tests are performed using log prices and return series. *, **, *** denote rejection of the null hypothesis at the $10 \%, 5 \%$, and $1 \%$ levels respectively. Models include an intercept but no deterministic trend. The following figure plots the historical weekly returns (\%) of the MSCI Frontier Markets and MSCI World indices over the studied period. 

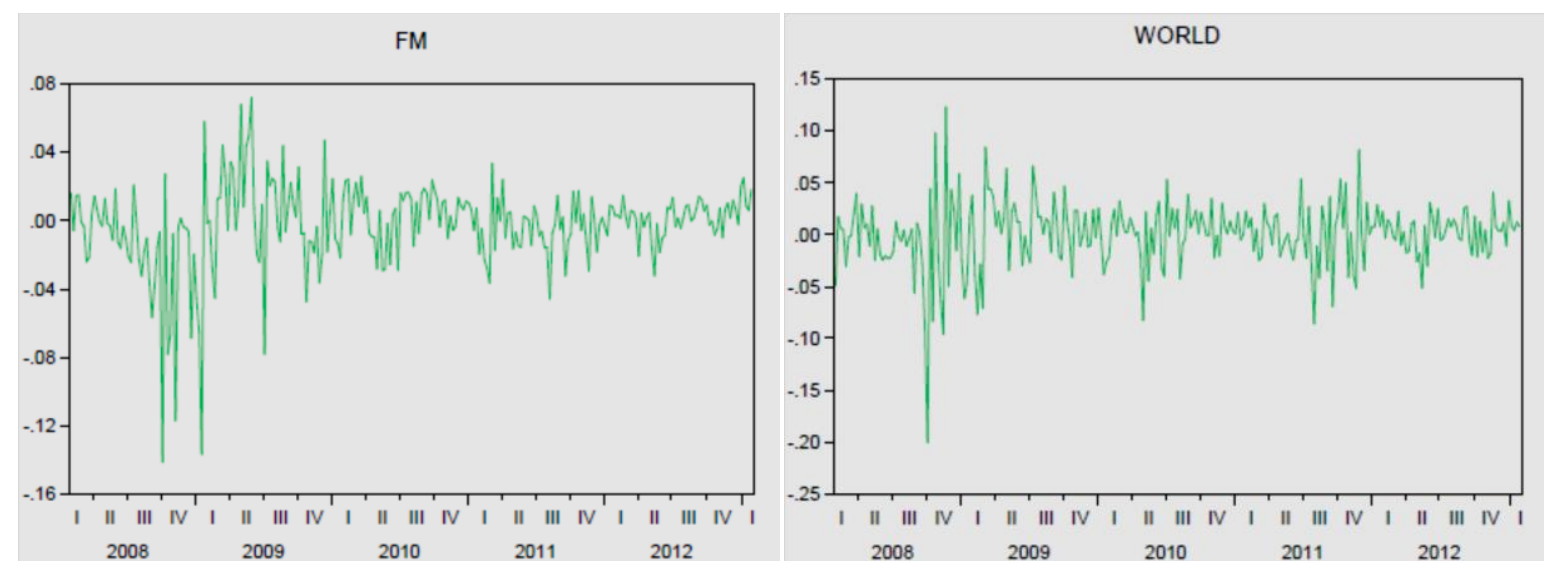

Figure 1: Weekly Returns of Broad Stock Market Indices (MSCI Frontier Markets and MSCI World)

Plots of each national equity market returns are enclosed in the appendix.

The following figure plots the historical weekly returns (\%) of crude oil (Brent) over the studied period.

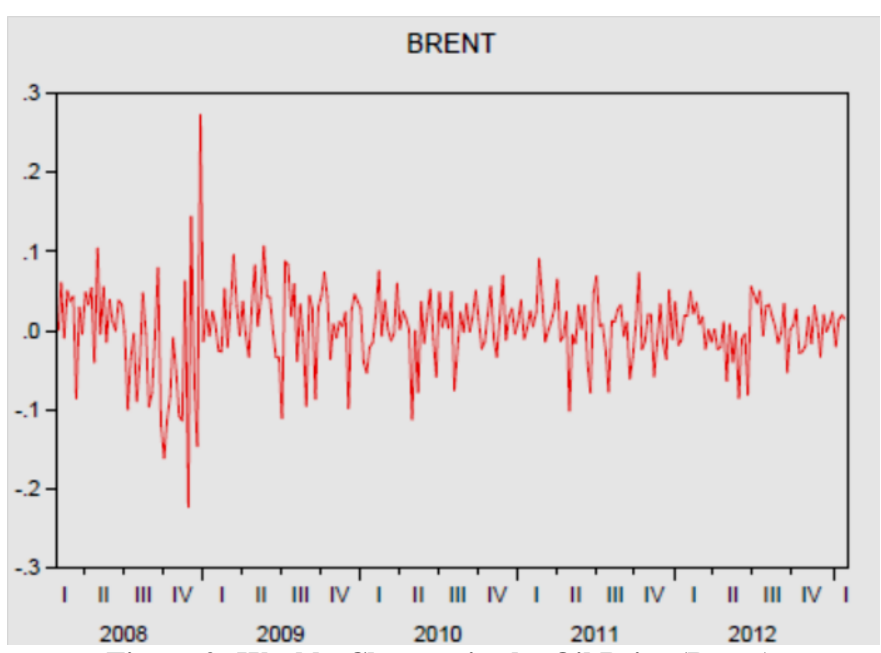

Figure 2: Weekly Changes in the Oil Price (Brent)

We can see that all markets experienced increased volatility during 2008 due to the financial crisis. Overall, frontier markets appear to be very volatile with countries such as Argentina, Romania, and Ukraine featuring mean annualized standard deviations above 40.0\%. However, the MSCI Frontier Markets index displays a mean weekly volatility of $2.53 \%$, which is actually lower than the MSCI World's (3.28\%). This tends to suggest that despite high individual volatilities, correlation between frontier equity markets is low.

Table 3 shows the correlation matrix for all considered markets. It tends to confirm the above statement, as correlations between frontier markets are indeed very low with an average value of $28.0 \%$. The average correlation coefficient between frontier markets and the Brent is $27.6 \%$ while average correlation between frontier markets and the MSCI World index amounts to $37.4 \%$. All these figures would tend to confirm the diversifying power of frontier markets stocks within a portfolio.

Correlations with oil are positive, suggesting that during the studied period, frontier stock markets moved together with oil prices. The highest correlation is between the MSCI World index and the oil market (0.5279). This result is expected because of the large proportion of American stocks within the index. The lowest significant correlation with oil market is obtained for Tunisia $(0.1313)$. 
Table 3: Correlation Matrix of Return Series (P-Values in Parentheses)

\begin{tabular}{|c|c|c|c|c|c|c|c|c|c|c|c|c|c|c|c|c|c|c|c|c|c|c|c|c|}
\hline & \begin{tabular}{|l|} 
ARG \\
\end{tabular} & BAH & BRENT & BUL & FM & JOR & KAZ & KEN & KUW & LEB & MAU & NIG & OMA & PAK & OAT & ROM & SAU & SLO & SRI & TUN & UAE & UKR & VIE & WORLD \\
\hline & 1.0000 & & & & & & & & & & & & & & & & & & & & & & & \\
\hline ARG & 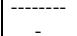 & & & & & & & & & & & & & & & & & & & & & & & \\
\hline BAH & 0.0955 & 1.0000 & & & & & & & & & & & & & & & & & & & & & & \\
\hline BRENT & 0.4347 & 0.1243 & 1.0000 & & & & & & & & & & & & & & & & & & & & & \\
\hline BKENI & (0.000) & $(0.045)$ & & & & & & & & & & & & & & & & & & & & & & \\
\hline BUL & {$\left[\begin{array}{l}0.4679 \\
(0.000)\end{array}\right.$} & $\begin{array}{l}0.2473 \\
0.000\end{array}$ & $\begin{array}{l}0.3510 \\
0.000\end{array}$ & 1.0000 & & & & & & & & & & & & & & & & & & & & \\
\hline FM & 0.4115 & 0.4137 & 0.3979 & 0.4843 & 1.0000 & & & & & & & & & & & & & & & & & & & \\
\hline $\mathrm{FNI}$ & (0.000) & $(0.000)$ & $(0.000)$ & $(0.000)$ & & & & & & & & & & & & & & & & & & & & \\
\hline JOR & $\mid \begin{array}{l}0.1724 \\
(0.005)\end{array}$ & $\begin{array}{l}0.2942 \\
(0.000)\end{array}$ & $\begin{array}{l}0.0689 \\
(0.267)\end{array}$ & $\begin{array}{l}0.3446 \\
(0.000)\end{array}$ & $\begin{array}{l}0.4710 \\
(0.000)\end{array}$ & 1.0000 & & & & & & & & & & & & & & & & & & \\
\hline & 0.5229 & 0.0927 & 0.4805 & 0.3893 & 0.3399 & 0.1630 & 1.0000 & & & & & & & & & & & & & & & & & \\
\hline KAZ & (0.000) & $(0.135)$ & $(0.000)$ & $(0.000)$ & $(0.000)$ & $(0.008)$ & & & & & & & & & & & & & & & & & & \\
\hline KEN & $\mid \begin{array}{l}0.2789 \\
(0.000)\end{array}$ & $\begin{array}{l}0.2260 \\
(0.000)\end{array}$ & $\begin{array}{l}0.1908 \\
(0.002)\end{array}$ & $\begin{array}{l}0.3140 \\
(0.000)\end{array}$ & $\begin{array}{l}0.3368 \\
(0.000)\end{array}$ & $\begin{array}{l}0.2931 \\
(0.000)\end{array}$ & $\begin{array}{l}0.2652 \\
(0.000)\end{array}$ & 1.0000 & & & & & & & & & & & & & & & & \\
\hline KUW & $\begin{array}{l}0.1969 \\
(0.001)\end{array}$ & $\begin{array}{l}0.3138 \\
(0.000)\end{array}$ & $\begin{array}{l}0.1601 \\
(0.010)\end{array}$ & $\begin{array}{l}0.1964 \\
(0.001)\end{array}$ & $\begin{array}{l}0.8057 \\
(0.000)\end{array}$ & $\begin{array}{l}0.2771 \\
(0.000\end{array}$ & $\begin{array}{l}0.1041 \\
(0.093)\end{array}$ & $\begin{array}{l}0.1583 \\
(0.010)\end{array}$ & 1.0000 & & & & & & & & & & & & & & & \\
\hline JFR & 0.3598 & 0.2351 & 0.2618 & 0.2337 & 0.3462 & 0.2122 & 0.2362 & 0.2023 & 0.1612 & 1.0000 & & & & & & & & & & & & & & \\
\hline LED & (0.000) & $(0.000)$ & $(0.000)$ & $(0.000)$ & $(0.000)$ & $(0.001)$ & $(0.000)$ & $(0.001)$ & $(0.009)$ & & & & & & & & & & & & & & & \\
\hline MAU & 0.2878 & 0.1912 & 0.3293 & 0.5230 & 0.4254 & 0.2800 & 0.2958 & 0.3307 & 0.1542 & 0.2046 & 1.0000 & & & & & & & & & & & & & \\
\hline & (0.000) & $(0.002)$ & $(0.000)$ & $(0.000)$ & $(0.000)$ & $(0.000)$ & $(0.000)$ & $(0.000)$ & (0.013) & $(0.001)$ & & & & & & & & & & & & & & \\
\hline NIG & 0.0237 & 0.1204 & 0.1184 & 0.1136 & 0.2873 & 0.1065 & 0.0845 & 0.0817 & -0.0102 & 0.1228 & 0.0614 & 1.0000 & & & & & & & & & & & & \\
\hline & & (0.052) & (0.156) & $(0.067)$ & $(0.000)$ & $(0.086)$ & $(0.174)$ & $(0.188)$ & $(0.870)$ & $(0.048)$ & $(0.323)$ & & & & & & & & & & & & & \\
\hline OMA & $\begin{array}{l}(0.000) \\
\end{array}$ & $\begin{array}{l}(0.000) \\
(0.284\end{array}$ & $(0.000)$ & $\begin{array}{l}\begin{array}{l}0.3589 \\
(0.000)\end{array}\end{array}$ & $\begin{array}{l}0.6493 \\
0\end{array}$ & 0.3500 & $\begin{array}{l}0.1446 \\
0.020\end{array}$ & $\begin{array}{l}0.3600 \\
0\end{array}$ & $\begin{array}{l}0.4353 \\
0.009\end{array}$ & $\begin{array}{l}0.1411 \\
(0.023)\end{array}$ & $\begin{array}{l}0.2645 \\
0\end{array}$ & $\begin{array}{l}0.1043 \\
0.093\end{array}$ & 1.0000 & & & & & & & & & & & \\
\hline PAK & 0.1092 & 0.1042 & 0.0699 & 0.0595 & 0.3412 & -0.0397 & 0.0867 & 0.0727 & 0.3256 & 0.0469 & 0.1557 & 0.0723 & 0.2457 & 1.0000 & & & & & & & & & & \\
\hline PAK & (0.078) & $(0.093)$ & $(0.261)$ & $(0.338)$ & $(0.000)$ & $(0.523)$ & $(0.163)$ & $(0.242)$ & $(0.000)$ & $(0.451)$ & $(0.012)$ & $(0.244)$ & $(0.000)$ & & & & & & & & & & & \\
\hline QAT & 0.2592 & 0.2707 & 0.2395 & 0.3639 & 0.7420 & 0.4510 & 0.1 & 0.29 & 9 & 0.2906 & 0.3 & 0.0876 & 0.5896 & 0.1181 & 1.0000 & & & & & & & & & \\
\hline & (0.000) & $(0.000)$ & $(0.000)$ & $(0.000)$ & $(0.000)$ & $(0.000)$ & (0. & $(0.000)$ & 10) & $(0.000)$ & $(0.000)$ & $(0.158)$ & $(0.000)$ & $(0.057)$ & & & & & & & & & & \\
\hline ROM & $\left(\begin{array}{l}0.4735 \\
(0.000)\end{array}\right.$ & $\begin{array}{l}0.2418 \\
(0.000)\end{array}$ & $\begin{array}{l}0.3788 \\
(0.000)\end{array}$ & $\begin{array}{l}0.5544 \\
(0.000)\end{array}$ & $\begin{array}{l}0.5709 \\
(0.000)\end{array}$ & $\begin{array}{l}0.2066 \\
(0.001)\end{array}$ & $\begin{array}{l}0.4789 \\
(0.000)\end{array}$ & $\begin{array}{l}0.3027 \\
(0.000)\end{array}$ & $\begin{array}{l}0.3312 \\
(0.000)\end{array}$ & $\begin{array}{l}0.2670 \\
(0.000)\end{array}$ & $\begin{array}{l}0.4684 \\
(0.000)\end{array}$ & $\begin{array}{l}0.2543 \\
(0.000)\end{array}$ & $\begin{array}{l}0.3059 \\
(0.000)\end{array}$ & $\begin{array}{l}0.1646 \\
(0.008)\end{array}$ & $\begin{array}{l}0.3638 \\
(0.000)\end{array}$ & 1.0000 & & & & & & & & \\
\hline SAU & $\begin{array}{l}0.2664 \\
(0.000)\end{array}$ & $\begin{array}{l}0.2402 \\
(0.000)\end{array}$ & $\begin{array}{l}0.1512 \\
(0.015)\end{array}$ & $\begin{array}{l}0.3921 \\
(0.000)\end{array}$ & $\begin{array}{l}0.6058 \\
(0.000)\end{array}$ & $\begin{array}{l}0.5031 \\
(0.000)\end{array}$ & $\begin{array}{l}0.0895 \\
(0.149)\end{array}$ & $\begin{array}{l}0.2687 \\
(0.000)\end{array}$ & $\begin{array}{l}0.3608 \\
(0.000)\end{array}$ & $\begin{array}{l}0.1999 \\
(0.001)\end{array}$ & $\begin{array}{l}0.3259 \\
(0.000)\end{array}$ & $\begin{array}{l}0.1440 \\
(0.020)\end{array}$ & $\begin{array}{l}0.5179 \\
(0.000)\end{array}$ & $\begin{array}{l}0.1696 \\
(0.000)\end{array}$ & $\begin{array}{l}0.6210 \\
(0.000)\end{array}$ & $\begin{array}{l}0.2662 \\
(0.000)\end{array}$ & 1.0000 & & & & & & & \\
\hline SLO & $\mid \begin{array}{l}0.5751 \\
(0.000)\end{array}$ & $\begin{array}{l}0.1366 \\
(0.027)\end{array}$ & $\begin{array}{l}0.4708 \\
(0.000)\end{array}$ & $\begin{array}{l}0.5838 \\
(0.000)\end{array}$ & $\begin{array}{l}0.5052 \\
(0.000)\end{array}$ & $\begin{array}{l}0.3389 \\
(0.000)\end{array}$ & $\begin{array}{l}0.4543 \\
(0.000)\end{array}$ & $\begin{array}{l}0.2856 \\
(0.000)\end{array}$ & $\begin{array}{l}0.2174 \\
(0.000)\end{array}$ & $\begin{array}{l}0.2893 \\
(0.000)\end{array}$ & $\begin{array}{l}0.5061 \\
(0.000)\end{array}$ & $\begin{array}{l}0.1272 \\
(0.040)\end{array}$ & $\begin{array}{l}0.3022 \\
(0.000)\end{array}$ & $\begin{array}{l}0.0996 \\
(0.108)\end{array}$ & $\begin{array}{l}0.3074 \\
(0.000)\end{array}$ & $\begin{array}{l}0.5753 \\
(0.000)\end{array}$ & $\begin{array}{l}0.3824 \\
(0.000)\end{array}$ & 1.0000 & & & & & & \\
\hline & 0.2073 & 0.1191 & 0.2418 & 0.2168 & 0.2686 & 0.1639 & 0.2487 & 0.2307 & 0.0884 & 0.0995 & 0.3208 & 0.1149 & 0.2181 & 0.0364 & 0.1351 & 0.2327 & 0.1830 & 0.3060 & 1.0000 & & & & & \\
\hline SRI & (0.001) & $(0.055)$ & $(0.000)$ & $(0.000)$ & $(0.000)$ & $(0.008)$ & $(0.000)$ & $(0.000)$ & $(0.154)$ & (0.109) & $(0.000)$ & $(0.064)$ & $(0.000)$ & $(0.559)$ & $(0.029)$ & $(0.000)$ & $(0.003)$ & $(0.000)$ & & & & & & \\
\hline TUN & 0.1853 & 0.0962 & 0.1313 & 0.2344 & 0.2442 & 0.1375 & 0.1292 & 0.2188 & 0.1490 & 0.0771 & 0.1793 & 0.0833 & 0.2043 & 0.0837 & 0.0771 & 0.2321 & 0.2208 & 0.2316 & 0.1279 & 1.0000 & & & & \\
\hline & $(0.003)$ & $(0.121)$ & $(0.034)$ & $(0.000)$ & $(0.000)$ & $(0.026)$ & $(0.037)$ & $(0.000)$ & $(0.016)$ & $(0.214)$ & $(0.004)$ & $(0.180)$ & $(0.001)$ & $(0.178)$ & $(0.214)$ & $(0.000)$ & $(0.000)$ & $(0.000)$ & $(0.039)$ & & & & & \\
\hline UAE & \begin{tabular}{|l|}
0.1950 \\
\end{tabular} & 0.3519 & 0.3156 & 0.3552 & 0.7654 & 0.4470 & 0.1912 & 0.2188 & 0.4763 & 0.2278 & 0.3670 & 0.1025 & 0.6552 & 0.2635 & 0.6333 & 0.3095 & 0.5874 & 0.3453 & 0.1921 & 0.1859 & 1.0000 & & & \\
\hline & (0.002) & $(0.000)$ & $(0.000)$ & $(0.000)$ & $(0.000)$ & $(0.000)$ & $(0.002)$ & $(0.000)$ & $(0.000)$ & $(0.000)$ & $(0.000)$ & $(0.099)$ & $(0.000)$ & $(0.000)$ & $(0.000)$ & $(0.000)$ & $(0.000)$ & $(0.000)$ & $(0.002)$ & $(0.003)$ & & & & \\
\hline UKR & 0.4063 & 0.1783 & 0.3340 & 0.4078 & 0.4157 & 0.2380 & 0.4850 & 0.2680 & 0.1776 & 0.2789 & 0.3191 & 0.1478 & 0.2779 & 0.1683 & 0.2798 & 0.4644 & 0.2829 & 0.3997 & 0.2706 & 0.1013 & 0.2787 & 1.0000 & & \\
\hline & & $(0.004)$ & $(0.000)$ & & & & & $(0.00$ & (0.004) & (0.000) & $(0.000)$ & & $(0.000)$ & & $(0.000)$ & $(0.000)$ & $(0.000)$ & $(0.000)$ & $(0.000)$ & & & 02780 & 10000 & \\
\hline VIE & $\begin{array}{l}(0.000) \\
\end{array}$ & $\begin{array}{l}.0 .010) \\
(0.140)\end{array}$ & $\begin{array}{l}.2869 \\
(0.000)\end{array}$ & $\begin{array}{l}(.3521 \\
(0.000)\end{array}$ & $\begin{array}{l}0.04480 \\
(0.000)\end{array}$ & $\begin{array}{l}.0 .19060 \\
(0.002)\end{array}$ & $\begin{array}{l}0.2083 \\
(0.001)\end{array}$ & $\begin{array}{l}.015360 \\
(0.012\end{array}$ & $\begin{array}{l}0.1344 \\
(0.030)\end{array}$ & $\begin{array}{l}0.1418 \\
(0.022)\end{array}$ & $\begin{array}{l}0.3448) \\
(0.000\end{array}$ & $\begin{array}{l}0.083 \\
(0.196)\end{array}$ & $\begin{array}{l}\begin{array}{l}0.2522 \\
(0.000)\end{array}\end{array}$ & $\begin{array}{l}0.0017 \\
(0.978)\end{array}$ & $\begin{array}{l}0.3238 \\
(0.000)\end{array}$ & $\begin{array}{l}\begin{array}{l}(299 / 5 \\
(0.000)\end{array}\end{array}$ & $\begin{array}{l}0.2755 \\
(0.00)\end{array}$ & $\begin{array}{l}0.3664 \\
(0.000)\end{array}$ & $\begin{array}{l}0.1141 \\
0.066\end{array}$ & 0.1240 & $\begin{array}{l}0.2798 \\
0\end{array}$ & $(0.000)$ & 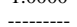 & \\
\hline WORLD & 0.6636 & 0.0614 & 0.5279 & 0.5801 & & 0.1730 & & 0.2757 & & & & 0.0912 & 0.2506 & & & 0.6474 & 0.2695 & 0.6534 & 0.1561 & 0.2580 & 0.2791 & 0.4382 & 0.3204 & 1.0000 \\
\hline & $(0.000)$ & $(0.323)$ & $(0.000)$ & $(0.000)$ & $(0.000)$ & $(0.005)$ & $(0.000)$ & $(0.000)$ & $(0.000)$ & $(0.000)$ & $(0.000)$ & $(0.142)$ & $(0.000)$ & $(0.046)$ & $(0.000)$ & $(0.000)$ & $(0.000)$ & $(0.000)$ & $(0.012)$ & $(0.000)$ & $(0.000)$ & $(0.000)$ & $(0.000)$ & \\
\hline
\end{tabular}


Before moving to the analysis of potential spillovers, it is interesting to examine short-term relationships between oil price changes and stock market returns. To do this, I perform a Granger causality test (for a lag equal to one) to examine the causal linkages between these series. Table 4 reports the results obtained.

Table 4: Results of the Granger Causality Tests (P-Values)

\begin{tabular}{lcc}
\hline & Oil to Stocks & Stocks to Oil \\
\hline ARG & 0.3174 & 0.8402 \\
BAH & 0.0151 & 0.1053 \\
BUL & 0.0126 & 0.1137 \\
FM & 0.0304 & 0.0711 \\
JOR & 0.0000 & 0.6258 \\
KAZ & 0.3884 & 0.7722 \\
KEN & 0.0065 & 0.4141 \\
KUW & 0.2452 & 0.1182 \\
LEB & 0.0065 & 0.2575 \\
MAU & 0.1574 & 0.1948 \\
NIG & 0.0324 & 0.3140 \\
OMA & 0.0001 & 0.7754 \\
PAK & 0.2880 & 0.6814 \\
QAT & 0.0092 & 0.0563 \\
ROM & 0.7098 & 0.2733 \\
SAU & 0.0000 & 0.3402 \\
SLO & 0.1322 & 0.6833 \\
SRI & 0.1778 & 0.1173 \\
TUN & 0.7459 & 0.5896 \\
UAE & 0.0037 & 0.0631 \\
UKR & 0.0009 & 0.1338 \\
VIE & 0.0585 & 0.3438 \\
WORLD & 0.9683 & 0.4878 \\
\hline
\end{tabular}

The results show that, in the short-run oil price shocks Granger-cause changes in stock market returns in, Jordan, Kenya, Lebanon, Oman, Qatar, Saudi Arabia, the UAE, and Ukraine (1\%) and to some extent in Bahrain, Bulgaria, the Frontier Markets index, Nigeria (5\%), and Viet Nam (10\%). There is also evidence of causality from the Frontier Markets Index, Qatar and the UAE stock markets to oil prices.

\section{METHODOLOGY}

The GARCH-type approach has received a special interest from almost all previous studies dealing with volatility modeling and forecasting of commodities prices. When the aim is to investigate volatility interdependence and transmission mechanisms among different time-series, multivariate settings such as the CCC-MGARCH model of Bollerslev (1990), the BEKK-GARCH model of Baba et al. (1990), or the DCC-MGARCH model of Engle (2002) are more relevant than univariate models. Empirical results reported in Hassan and Malik (2007) and Agnolucci (2009), among others, confirm the superiority of these models and show that they are able to satisfactorily capture the stylized facts of the commodity-price conditional volatility and the dynamics of volatility interaction.

The first step in the bivariate GARCH methodology is to specify the mean equation. Accordingly, the mean equations for oil and stock return series are given by:

$r_{o}=\mu_{o}+\phi_{o} r_{o-1}+\varepsilon_{o}$

$r_{s}=\mu_{s}+\phi_{s} r_{s-1}+\varepsilon_{s}$

where $r_{o}$ and $r_{s}$ are the returns between time $\mathrm{t}-1$ and $\mathrm{t}$ on oil and stock series respectively, $r_{o-1}$ and $r_{s-1}$ are lagged returns on oil and stock series, $\mu_{o}$ and $\mu_{s}$ are long term drift coefficients, and $\varepsilon_{o}$ and $\varepsilon_{s}$ are error terms. Various models were tested to specify the mean equation (ARMA type) but the coefficients were not statistically significant so I ended up using the classical first order autoregressive model. 
In this paper, we use a bivariate GARCH (Generalized Auto Regressive Conditionally Heteroscedastic) model to estimate the conditional variance of oil and stock index returns. In addition, I employ the BEKK (named after Baba, Engle, Kraft, and Kroner, 1990) parameterization of the multivariate GARCH model, which does not impose the restriction of constant correlation among variables over time. The model addresses the difficulty with $\mathrm{VECH}$ of ensuring that the $\mathrm{H}$ matrix is always positive definite by incorporating quadratic forms.

The BEKK parameterization for the bivariate $\operatorname{GARCH}(1,1)$ model is represented by:

$H_{t}=C C^{\prime}+A \varepsilon_{t-1} \varepsilon_{t-1}^{\prime} A^{\prime}+B H_{t-1} B^{\prime}$

where the individual elements for $\mathrm{C}, \mathrm{A}$ and $\mathrm{B}$ matrices are given as:

$\mathrm{A}=\left[\begin{array}{cc}\beta_{o} & \beta_{s o} \\ \beta_{o s} & \beta_{s}\end{array}\right] \quad \mathrm{B}=\left[\begin{array}{cc}\delta_{o} & \delta_{s o} \\ \delta_{o s} & \delta_{s}\end{array}\right] \quad \mathrm{C}=\left[\begin{array}{cc}\alpha_{o} & \alpha_{s o} \\ 0 & \alpha_{s}\end{array}\right]$

where $H_{t}$ is the conditional variance matrix, $\mathrm{C}$ is an upper triangular matrix of parameters, $\mathrm{B}$ is a 2 x 2 matrix of parameters which depicts the extent to which current levels of conditional variances are related to past conditional variances, and A is a $2 \times 2$ matrix of parameters that measures the extent to which conditional variances are correlated with past squared errors. In other words, A captures the effect of shocks or events on volatility. The positive definiteness of the covariance matrix is ensured owing to the quadratic nature of the terms on the equation's right hand side.

Expanding the conditional variance for each equation in the bivariate BEKK-GARCH(1,1) model gives:

$$
\begin{aligned}
& h_{o}^{2}=\alpha_{o}+\beta_{o}^{2} \varepsilon_{o-1}^{2}+2 \beta_{o} \beta_{s o} \varepsilon_{s-1} \varepsilon_{o-1}+\beta_{s o}^{2} \varepsilon_{s-1}^{2}+\delta_{o}^{2} h_{o-1}^{2}+2 \delta_{o} \delta_{s o} h_{s o-1}+\delta_{s o}^{2} h_{s-1}^{2} \\
& h_{s o}=\alpha_{s o}+\beta_{s} \beta_{o} \varepsilon_{s-1}^{2}+\left(\beta_{o s} \beta_{s o}+\beta_{s} \beta_{o}\right) \varepsilon_{s-1} \varepsilon_{o-1}+\beta_{o s} \beta_{o} \varepsilon_{o-1}^{2}+\delta_{s} \delta_{o} h_{s-1}^{2}+\left(\delta_{o s} \delta_{s o}+\delta_{s} \delta_{o}\right) h_{s o-1}+\delta_{o s} \delta_{o} h_{o-1}^{2} \\
& h_{s}^{2}=\alpha_{s}+\beta_{s}^{2} \varepsilon_{s-1}^{2}+2 \beta_{s} \beta_{o s} \varepsilon_{s-1} \varepsilon_{o-1}+\beta_{o s}^{2} \varepsilon_{o-1}^{2}+\delta_{s}^{2} h_{s-1}^{2}+2 \delta_{s} \delta_{o s} h_{s o-1}+\delta_{o s}^{2} h_{o-1}^{2}
\end{aligned}
$$

As can be seen, the conditional variance of the stock market (respectively oil market) depends not only on its own past variances and innovations, but also on those of the oil market (respectively stock market). This feature permits the direct transmission of volatility and shocks from one market to another. Overall, this model allows me to capture both return and volatility spillover effects between stock and oil markets.

Under the assumption of conditional normality, the parameters of a multivariate GARCH model can be estimated by maximizing the log-likelihood function:

$$
\begin{aligned}
& \operatorname{Max} \log L_{T}(\theta)=\sum_{t=1}^{T} l_{t}(\theta) \\
& l_{t}(\theta)=-\frac{T N}{2} \log (2 \pi)-\frac{1}{2} \sum_{t=1}^{T}\left(\log \left|H_{t}\right|+\varepsilon_{t}^{\prime} H_{t}^{-1} \varepsilon_{t}\right)
\end{aligned}
$$

where $\theta$ denotes all the unknown parameters to be estimated, $\mathrm{N}$ is the number of series and $\mathrm{T}$ is the number of observations.

\section{EMPIRICAL RESULTS}

In this section, we present in Table 5 the empirical results from estimating the bivariate BEKKGARCH $(1,1)$ model for all pairs of oil and stock market returns and we discuss the extent of volatility transmission. There are no reported data for Bulgaria and Romania as the model was unable to converge for these two markets. The symbols *,** and *** indicate significance at $10 \%, 5 \%$, and $1 \%$ levels respectively. 
Taking a close look at mean equations, we find that the one-period lagged oil returns, denoted by $\phi_{o}$ coefficients, never affect current oil returns significantly. For stock returns, autoregressive coefficients $\phi_{s}$ are usually not significant, which is in line with the efficient markets hypothesis. Exceptions are the Frontier Markets Index, Mauritius, and Ukraine, all at the $1 \%$ level. This finding tends to suggest that for these three markets, past stock realizations have some predictive power on current stock returns, which can be explained by the emerging nature of these markets (e.g., a low level of liquidity and smaller capitalizations). The constant terms for the stock returns mean equations are only significant for Kenya and the MSCI World index (both at the 1\% level).

Table 5: Estimates of BEKK-GARCH(1,1) Model for Oil and Stock Returns

\begin{tabular}{|c|c|c|c|c|c|c|c|}
\hline & ARG & BAH & FM & JOR & KAZ & KEN & KUW \\
\hline \multicolumn{8}{|c|}{ Panel A: Conditional Mean Estimates } \\
\hline$\mu_{o}$ & 0.0049 & 0.0070 & 0.0032 & 0.0044 & 0.0041 & 0.0039 & 0.0037 \\
\hline$\phi_{o}$ & 0.0208 & 0.0742 & -0.0645 & -0.0104 & 0.0260 & 0.0202 & 0.0023 \\
\hline$\mu_{s}$ & 0.0003 & -0.0014 & 0.0011 & -0.0016 & 0.0013 & $0.0046 * * *$ & -0.0012 \\
\hline$\phi_{s}$ & -0.0774 & 0.0534 & $0.1388 * * *$ & -0.0999 & 0.0242 & 0.1069 & 0.0698 \\
\hline \multicolumn{8}{|c|}{ Panel B: Conditional Variance-Covariance Estimates } \\
\hline$\alpha_{o}$ & $0.0287 * * *$ & 0.0129 & 0.0122 & 0.0113 & 0.0112 & 0.0083 & 0.0118 \\
\hline$\alpha_{\text {so }}$ & 0.0380 & 0.0082 & -0.0003 & -0.0008 & 0.0084 & -0.0088 & 0.0001 \\
\hline$\alpha_{s}$ & 0.0000 & 0.0000 & 0.0000 & 0.0024 & 0.0083 & 0.0000 & 0.0000 \\
\hline$\beta_{o}$ & 0.4056 & -0.0958 & 0.0494 & -0.0846 & 0.0176 & 0.0472 & $-0.2928 * * *$ \\
\hline $\boldsymbol{\beta}_{\text {os }}$ & $0.6972 * * *$ & $-0.2349 * * *$ & $-0.1690 * * *$ & 0.1164 & 0.1964 & $0.1780 * *$ & -0.0196 \\
\hline$\beta_{\text {so }}$ & 0.1187 & 0.2258 & $-0.9650 * *$ & 0.0220 & 0.2339 & 0.3752 & -0.0394 \\
\hline$\beta_{s}$ & -0.1441 & $-0.8011 * * *$ & 0.2192 & $0.3437 * * *$ & 0.3147 & $0.3456^{* * *}$ & $0.2808 * *$ \\
\hline$\delta_{o}$ & $0.7030 * * *$ & $-0.8600 * * *$ & 0.0547 & -0.1990 & $-1.0746 * * *$ & $0.7539 * * *$ & $-0.4019 * *$ \\
\hline$\delta_{o s}$ & -0.4561 & 0.0960 & $0.3972 * * *$ & $0.4963 * *$ & $-0.2840 * *$ & $0.4195 * * *$ & $0.5087 * * *$ \\
\hline$\delta_{\text {so }}$ & -0.1529 & -0.4031 & $-2.0541 * * *$ & $1.1711 * * *$ & $0.3429 * * *$ & -0.9459 & $-1.1751 * * *$ \\
\hline \multirow[t]{2}{*}{$\delta_{s}$} & 0.6340 & $-0.6055^{* * *}$ & $-0.7568 * * *$ & 0.2202 & $-0.6911 * * *$ & $0.5384 * * *$ & $-0.6911 * * *$ \\
\hline & LEB & MAU & NIG & OMA & PAK & QAT & SAU \\
\hline \multicolumn{8}{|c|}{ Panel A: Conditional Mean Estimates } \\
\hline$\mu_{o}$ & 0.0042 & 0.0032 & 0.0027 & 0.0054 & 0.0022 & 0.0017 & 0.0022 \\
\hline$\phi_{o}$ & -0.0181 & -0.0111 & 0.0317 & -0.0475 & 0.0095 & -0.0021 & -0.0116 \\
\hline$\mu_{s}$ & -0.0012 & 0.0011 & 0.0007 & -0.0001 & $0.0024 *$ & 0.0019 & 0.0014 \\
\hline$\phi_{s}$ & -0.0069 & $0.1981 * * *$ & 0.1099 & -0.0303 & $0.1130 *$ & 0.0888 & -0.0298 \\
\hline \multicolumn{8}{|c|}{ Panel B: Conditional Variance-Covariance Estimates } \\
\hline$\alpha_{o}$ & 0.0096 & $0.01193 * *$ & 0.0097 & $0.0119 * *$ & $0.0067 * *$ & 0.0162 & 0.0082 \\
\hline$\alpha_{\text {so }}$ & 0.0059 & 0.0012 & -0.0039 & -0.0001 & -0.0050 & 0.0004 & 0.0036 \\
\hline$\alpha_{s}$ & 0.0000 & 0.0000 & 0.0000 & 0.0000 & 0.0000 & 0.0000 & 0.0102 \\
\hline$\beta_{o}$ & $0.2077 * *$ & 0.0158 & -0.2578 & 0.0674 & $0.1227 * * *$ & -0.3698 & -0.0813 \\
\hline$\beta_{\text {os }}$ & 0.0630 & 0.0789 & $0.1450 * *$ & $0.1852 * *$ & 0.1612 & -0.0872 & 0.1501 \\
\hline$\beta_{\text {so }}$ & 0.1460 & 0.0943 & 0.3001 & $0.4959 * * *$ & 0.0876 & 0.2864 & 0.1799 \\
\hline$\beta_{s}$ & $0.3959 * * *$ & $0.4275^{* * *} *$ & 0.2647 & 0.1231 & $-0.7228 * * *$ & 0.3821 & $-0.6519 * * *$ \\
\hline$\delta_{o}$ & $0.9300 * * *$ & $-0.4084 * * *$ & $0.8890 * * *$ & $0.5165 * * *$ & -0.9943 & $-0.3150 * * *$ & $-0.8973 * * *$ \\
\hline$\delta_{o s}$ & $-0.0849 * * *$ & $0.4506 * * *$ & 0.3465 & $-0.3910 * * *$ & $-0.1515 * * *$ & -0.6492 & 0.0394 \\
\hline$\delta_{\text {so }}$ & 0.0923 & -1.2326 & 0.1304 & $1.0783 * * *$ & 0.3999 & 1.1078 & -0.3258 \\
\hline \multirow[t]{2}{*}{$\delta_{s}$} & $0.9039 * * *$ & $-0.7103 * * *$ & $-0.9098 * * *$ & $0.8439 * * *$ & 0.7825 & $-0.2149 * *$ & $-0.7324 * * *$ \\
\hline & SLO & SRI & TUN & UAE & UKR & VIE & WORLD \\
\hline \multicolumn{8}{|c|}{ Panel A: Conditional Mean Estimates } \\
\hline$\mu_{o}$ & 0.0032 & 0.0025 & 0.0028 & 0.0056 & 0.0036 & 0.0034 & 0.0066 \\
\hline$\phi_{o}$ & 0.0102 & -0.0333 & 0.0122 & -0.0498 & -0.0123 & -0.0395 & 0.0171 \\
\hline$\mu_{s}$ & -0.0033 & -0.0003 & 0.0008 & 0.0024 & -0.0055 & -0.0017 & $0.0041 * * *$ \\
\hline$\phi_{s}$ & 0.0184 & 0.1496 & 0.0167 & 0.0138 & $0.2076^{* * *}$ & 0.0612 & -0.1053 \\
\hline \multicolumn{8}{|c|}{ Panel B: Conditional Variance-Covariance Estimates } \\
\hline$\alpha_{o}$ & -0.0086 & 0.0183 & 0.0036 & $0.0165 * * *$ & 0.0227 & 0.0069 & 0.0189 \\
\hline$\alpha_{\text {so }}$ & -0.0133 & -0.0025 & 0.0045 & 0.0009 & 0.0307 & -0.0172 & -0.0032 \\
\hline$\alpha_{s}$ & 0.0232 & 0.0000 & 0.0000 & 0.0000 & 0.0000 & 0.0000 & 0.0000 \\
\hline$\beta_{o}$ & 0.1772 & $0.3630 * * *$ & 0.2345 & 0.0449 & 0.4033 & 0.1350 & 0.2555 \\
\hline $\boldsymbol{\beta}_{o s}$ & 0.1453 & 0.0624 & 0.0573 & 0.0966 & 0.0496 & -0.0903 & 0.1114 \\
\hline
\end{tabular}




\begin{tabular}{|c|c|c|c|c|c|c|c|}
\hline $\boldsymbol{\beta}_{\mathrm{c}}$ & 0.1315 & 0.5035 & 0.1454 & $-0.1574 * * * *$ & -0.1162 & -0.0362 & 0.2926 \\
\hline$\beta_{s}$ & 0.5478 & -0.1027 & $0.3215 * * *$ & $0.3818 * * *$ & 0.4308 & $0.5194 * * *$ & 0.5199 \\
\hline$\delta_{o}$ & $0.7986 * * *$ & 0.0941 & $-0.8481 * * *$ & $-0.7835 * * *$ & $-0.8177 * * *$ & $0.9189 * * *$ & $0.8759 * *$ \\
\hline$\delta_{o s}$ & 0.2133 & $0.7514 * * *$ & -0.2658 & $0.2189 * * *$ & 0.1297 & -0.0023 & $0.5009 * * *$ \\
\hline$\delta_{\text {so }}$ & 0.3444 & 0.9492 & -0.6788 & $-0.3173 * * *$ & 0.4256 & 0.1530 & $-1.2659 * * *$ \\
\hline$\delta_{s}$ & 0.0142 & 0.0015 & $0.8402 * * *$ & $-0.9498 * * *$ & 0.6506 & $0.8071 * * *$ & 0.0303 \\
\hline
\end{tabular}

Estimates of ARCH and GARCH coefficients, which capture shock dependence and volatility persistence in the conditional variance equations, are statistically significant at conventional levels in many cases. For the stock market segment, the sensitivity to past own conditional volatility $\left(\delta_{s}\right)$ appears to be significant for fourteen series at conventional levels.

The results also suggest that the current value of conditional volatility of stock returns often depends on past unexpected shocks affecting return dynamics since the associated coefficients $\left(\beta_{s}\right)$ are statistically significant in eleven cases. Moreover, the large magnitude of GARCH coefficients suggests that conditional volatility tends to fluctuate gradually over time.

Oil price volatility behaves similarly to stock market volatility as sensitivity to past own conditional volatility $\left(\delta_{o}\right)$ appears to be significant for all series at conventional levels except for Jordan, Sri Lanka, Pakistan, and the MSCI Frontier Markets Index. Similar conclusions can be drawn regarding sensitivity to past own shocks $\left(\beta_{o}\right)$ for four countries, namely Kuwait, Lebanon, Pakistan, and Sri Lanka.

Regarding volatility transmission between oil and stock markets, there can be observed that conditional volatility of stock markets is sometimes affected by innovations in the oil market as indicated by the significance of the $\beta_{\text {os }}$ coefficients for Argentina, Bahrain, Kenya, Nigeria, Oman, and the MSCI Frontier Markets Index. This seems to suggest that a shock originating from the oil market generally has an impact on stock market volatility in these markets. This is not illogical given the economic structure of these countries, in which the energy (Argentina, Bahrain, Nigeria, Oman, FM index) and financials sectors (Kenya, FM index) account for big proportions of GDP. The $\beta_{o s}$ highest value is found for Argentina. This can be explained by the importance of the manufacturing sector within its economy ( $20 \%$ of the countries' GDP). Moreover, the country is an oil producer and petroleum products account for about $10 \%$ of its exports.

In addition, there is evidence suggesting that past volatility of oil markets affect current stock market volatility as indicated by significant $\delta_{o s}$ coefficients for twelve series. The highest $\delta_{o s}$ coefficient measuring the volatility spillover from oil to stock markets is found for the MSCI Frontier Markets Index, which is not surprising given the importance of oil-centered economies within the index with Kuwait, Qatar, Nigeria, and the United Arab Emirates accounting for $65.29 \%$ of its composition. Moreover, if we take a look at the index sectorial composition, we see that Financials, Industrials and Energy account for $69.58 \%$ of its constituents. These are sectors for which we usually document significant volatility spillovers with oil for fundamental reasons (oil is an input for Industrials, and has an impact on consumer and investor confidence, as well as on monetary policy, thus affecting financials). The second highest coefficient is found for Jordan, which despite not being an oil exporting country is heavily dependent on oil for its transportation and industrial sector (approximately one fifth of its GDP), both major consumers of petroleum products.

Interestingly, the statistical significance of the $\beta_{\text {so }}$ (three cases) and $\delta_{s o}$ (seven cases) coefficients in the conditional variance equation for oil returns suggests that the oil market volatility is influenced by changes (shocks and volatility) that occurred in some frontier stock markets. The highest $\delta_{s o}$ (volatility transmission) coefficient is found for the MSCI World index while the highest $\beta_{\text {so }}$ (shock transmission) coefficient is found for the MSCI Frontier Markets index. Strangely, $\delta_{s o}$ is also significant for Jordan, which is unexpected. Further studies would be needed to investigate this relationship but this probably results from its geographical situation. Indeed, immediate proximity with countries such as Iraq or Saudi Arabia probably induces comovements between stock markets of these countries, which appear as spillovers. 
These findings are interesting as they suggest wide bi-directional transmission of both shock and volatility between oil and some frontier stock markets, but also between oil and the MSCI World Index, which is not illogical. Indeed, Arouri et al. (2011) demonstrated that the United States, which represents the major part (53.9\%) of the MSCI World Index composition, feature this bidirectional relationship between oil and stock markets. Moreover, the volatility spillover effect is often strong (usually significant at the $1 \%$ level). Regarding the direction of transmission, $\beta_{o s}$ and $\delta_{o s}$ coefficients with statistical significance are more numerous than their $\beta_{s o}$ and $\delta_{s o}$ counterparts, suggesting that spillovers from oil to stock markets are more usual than spillovers going from stocks to the oil market, which is in line with the majority of previous studies.

Summarizing all, direct volatility transmission between oil and stock returns is significantly present for many of the studied markets, including the MSCI World Index. Regarding the transmission's direction, shock and volatility spillovers happen more often running from oil to stock markets than from stock markets to the oil market, but there are divergences between countries, which is something to be expected given the heterogeneity of frontier market economies.

Finally, it is important to note that the set of data analyzed in this paper pertains to a very turbulent period in financial markets and that consequently, systematic factors may have played a role and biased the spillover results to some extent as spillovers usually increase during crisis periods under the effects of important financial instability and economic uncertainties.

\section{CONCLUSION}

This paper examined the transmission of shocks and volatility between oil prices and Frontier stock markets. The statistical model includes a parameterization of the conditional variance-covariance of oil price changes and stock returns, specifically the bivariate BEKK-GARCH $(1,1)$ model, which enables the analysis of spillovers in both returns and conditional variance. Aggregate stock market data representing twenty-three frontier markets, as well as two broad equity indices (MSCI World and MSCI Frontier Markets) are used. My analysis uses weekly data from February 8, 2008 to February 1, 2013, and provides estimates of the extent to which shocks and volatility are transmitted between oil returns and the returns of Frontier equity markets (+ the MSCI World Index). My results suggest significant volatility interaction between oil and some frontier stock markets. Moreover, the spillover effect appears to be bidirectional in many markets, which is a characteristic that differs from what has been found for developed stock markets where the transmission is usually unidirectional (from oil to stock markets). However, empirical results show that the spillovers run more often from oil to stock markets.

The findings of the study offer several avenues for future research. First, the link between oil and frontier stock markets should eventually be examined over an extended period of time. Second, further evidence drawn from a deeper decomposition of spillovers (spillover, interdependence, comovements and independence) should be produced to examine the robustness of the findings. These would require more elaborate models (MCMS). Finally, hedging effectiveness could be analyzed for these markets in order to compute optimal weights and hedge ratios for oil-stock portfolio holdings. Overall, this study is of particular interest for portfolio management since it offers insight into markets that still provide valuable opportunities for portfolio diversification. In all, this paper improves our knowledge of how stock markets relate to oil prices.

\section{AUTHOR INFORMATION}

Mathieu Gomes is a commodity index structuring analyst at BNP Paribas. He holds a Master's degree in financial markets and a Master's degree in management from the Université d'Auvergne, Clermont-Ferrand, France. His research interests include commodities, investment strategies, and financial economics. E-mail: mathieu.gomes@gmail.com

Dr. Anissa Chaibi is a researcher at IPAG Business School, France. Her research areas are development economics, finance, and econometrics. Her most recent articles are published in refereed journals such as Economic Modelling, Energy Policy and Journal of Applied Business Research. E-mail: anissa.chaibi@ipag.fr (Corresponding author) 


\section{REFERENCES}

1. Adelman, M. A. (1993). The economics of petroleum supply: Papers 1962-1993. Massachusetts Institute of Technology.

2. Agnolucci, P. (2009). Volatility In crude oil futures: A comparison of the predictive ability of GARCH and implied volatility models. Energy Economics, 31, 316-321.

3. Agren, M. (2006). Does oil price uncertainty transmit to stock markets? (Working Paper 2006, p. 23). Department of Economics/Uppsala University.

4. Arouri, M., \& Nguyen, D. K. (2010). Oil prices, stock markets and portfolio investment: Evidence from sector analysis in europe over the last decade. Energy Policy, 38, 4528-4539.

5. Arouri, M., Bellalah, M., \& Nguyen, D. K. (2011). Further evidence on the response of stock prices in GCC countries to oil price shocks. International Journal of Business, 16.

6. Arouri, M., Jouini, J., \& Nguyen, D. K. (2011). Volatility spillovers between oil prices and stock sector returns: Implications for portfolio management. Journal of International Money and Finance, 30, 13871405.

7. Arouri, M., Jouini, J., \& Nguyen, D. K. (2012). On the impact of oil price fluctuations on european equity markets: Volatility spillover and hedging effectiveness. Energy Economics, 34, §11-617.

8. Baba, Y., Engle, R.F., Kraft, D., \& Kroner, K. (1990). Multivariate simultaneous generalized ARCH . (Unpublished Manuscript). University of California, San Diego.

9. $\quad$ Berger, D., Pukthuanthong, K., \& Yang, J. J. (2011). International diversification with frontier markets. Journal of Financial Economics, 101, 227-242.

10. Boyer, M. M., \& Filion, D. (2007). Common and fundamental factors in stock returns of Canadian oil and gas companies. Energy Economics, 29, 428-453.

11. Chang, C., McAleer, M., \& Tansuchat, R. (2012). Conditional correlations and volatility spillovers between crude oil and stock index returns. North American Journal of Economics and Finance.

12. Ciner, C. (2001). Energy shocks and financial markets: Nonlinear linkages. Studies in Non-Linear Dynamics and Econometrics, 5, 203-212.

13. El-Sharif, I., Brown, D., Burton, B., Nixon, B., \& Russel, A. (2005). Evidence on the nature and extent of the relationship between oil and equity value in UK. Energy Economics, 27, 819-830.

14. Engle, R. F., \& Kroner, K. F. (1995). Multivariate simultaneous generalized ARCH. Econometric Theory, $11,122-150$.

15. Engle, R. F. (2002). Dynamic conditional correlation: A simple class of multivariate GARCH models. Journal of Business and Economic Statistics, 20, 339-350.

16. Gisser, M., \& Goodwin, T. H. (1986). Crude oil and the macroeconomy: Tests of some popular notions: Note. Journal of Money, Credit and Banking, 18(1), 95-103.

17. Hamilton, J. D. (1983). Oil and the macroeconomy since World War II. Journal of Political Economy, 91(2), 228-248.

18. Hassan, H., \& Malik, F. (2007). Multivariate GARCH model of sector volatility transmission. Quarterly Review of Economics and Finance, 47, 470-480.

19. Hooker, M. A. (1996). What happened to the oil price-macroeconomy relationship? Journal of Monetary Economics, 38, 195-213.

20. Huang, R. D., Masulis R. W., \& Stoll, H. R. (1996). Energy shocks and financial markets. Journal of Futures Markets, 16, 1-27.

21. Jayasuriya, S., \& Shambora, W. (2009). Oops, we should have diversified! Applied Financial Economics, $19,1779-1785$.

22. Jones, C. M., \& Kaul G. (1996). Oil and the stock markets. Journal of Finance, 51, 463-491.

23. Khalifa, A., Hammoudeh, S., \& Otranto, E. (2012). Volatility Spillover, interdependance, comovements across the GCC, oil and US markets and portfolio management strategies in a regime-changing environment. (Working Paper).

24. Malik, F., \& Hammoudeh, S. (2005). Shock and volatility transmission in the oil, US and Gulf equity markets. International Review of Economics and Finance, 16, 357-368.

25. Malik, F., \& Ewing, B. T. (2009). Volatility transmission between oil prices and equity sector returns. International Review of Financial Analysis, 18, 95-100. 
26. Mork, K. A. (1989). Oil and the macroeconomy when prices go up and down: An extension of hamilton's results. Journal of Political Economy, 97(3), 740-744.

27. Park, J., \& Ratti, R. A. (2008). Oil price shocks and stock markets in the US and 13 European Countries. Energy Economics, 30, 2587-2608.

28. Sadorsky, P. (1999). Oil price shocks and stock market activity. Energy Economics, 21, 449-469.

29. Speidell, L., \& Krohne, A. (2007). The case for frontier equity markets. Journal of Investing, 16, $12-22$. 


\section{Weekly Returns of National Equity Markets}
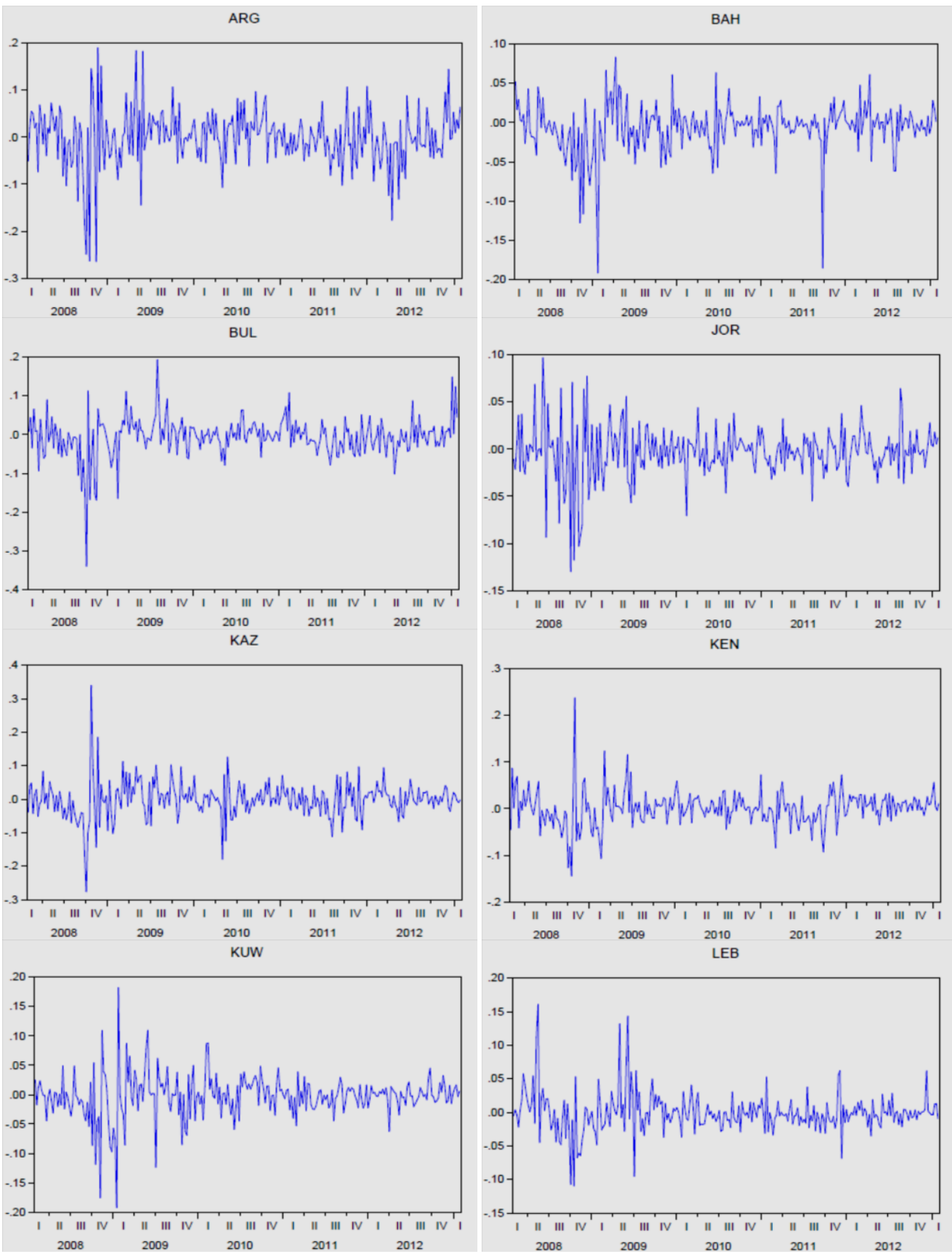

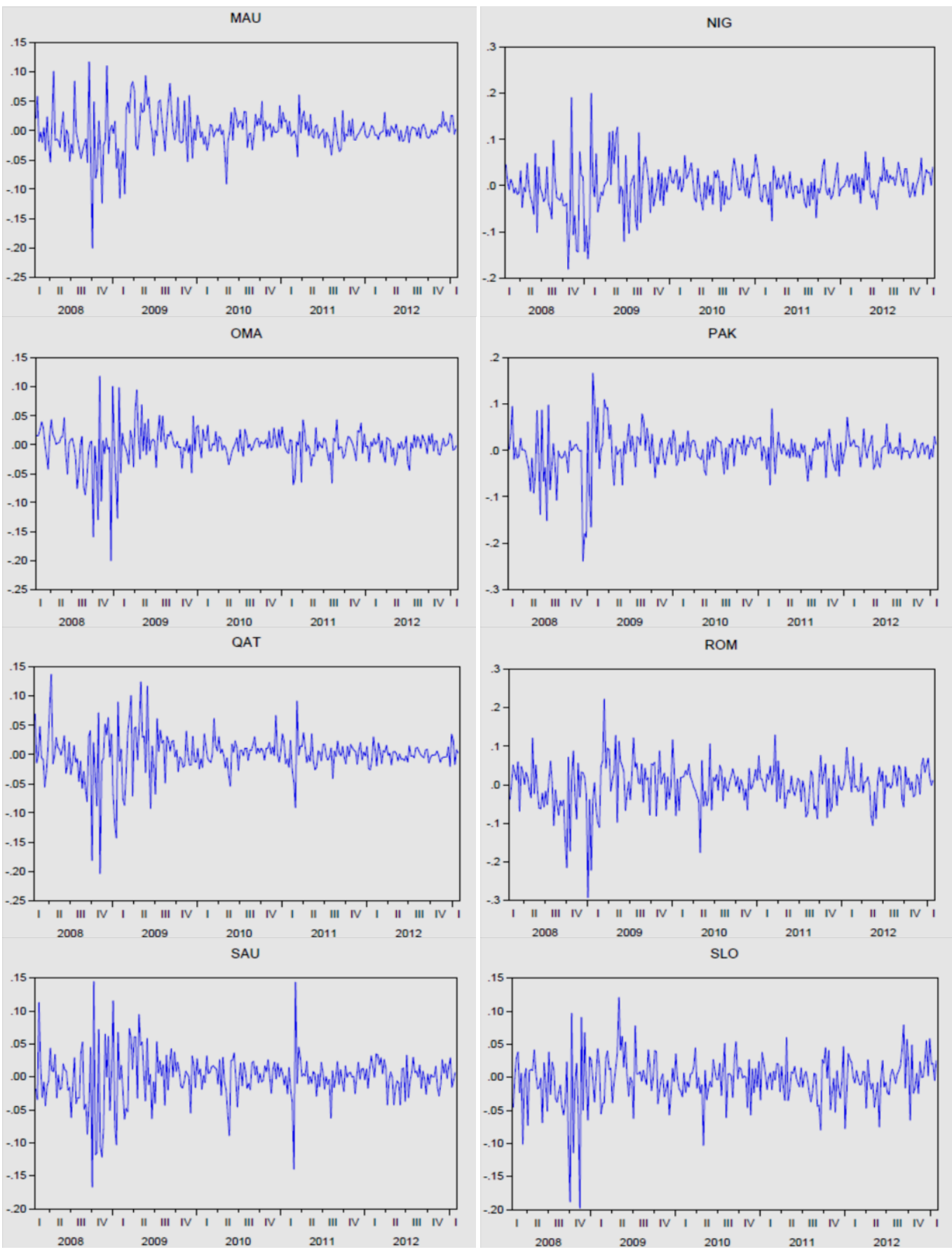

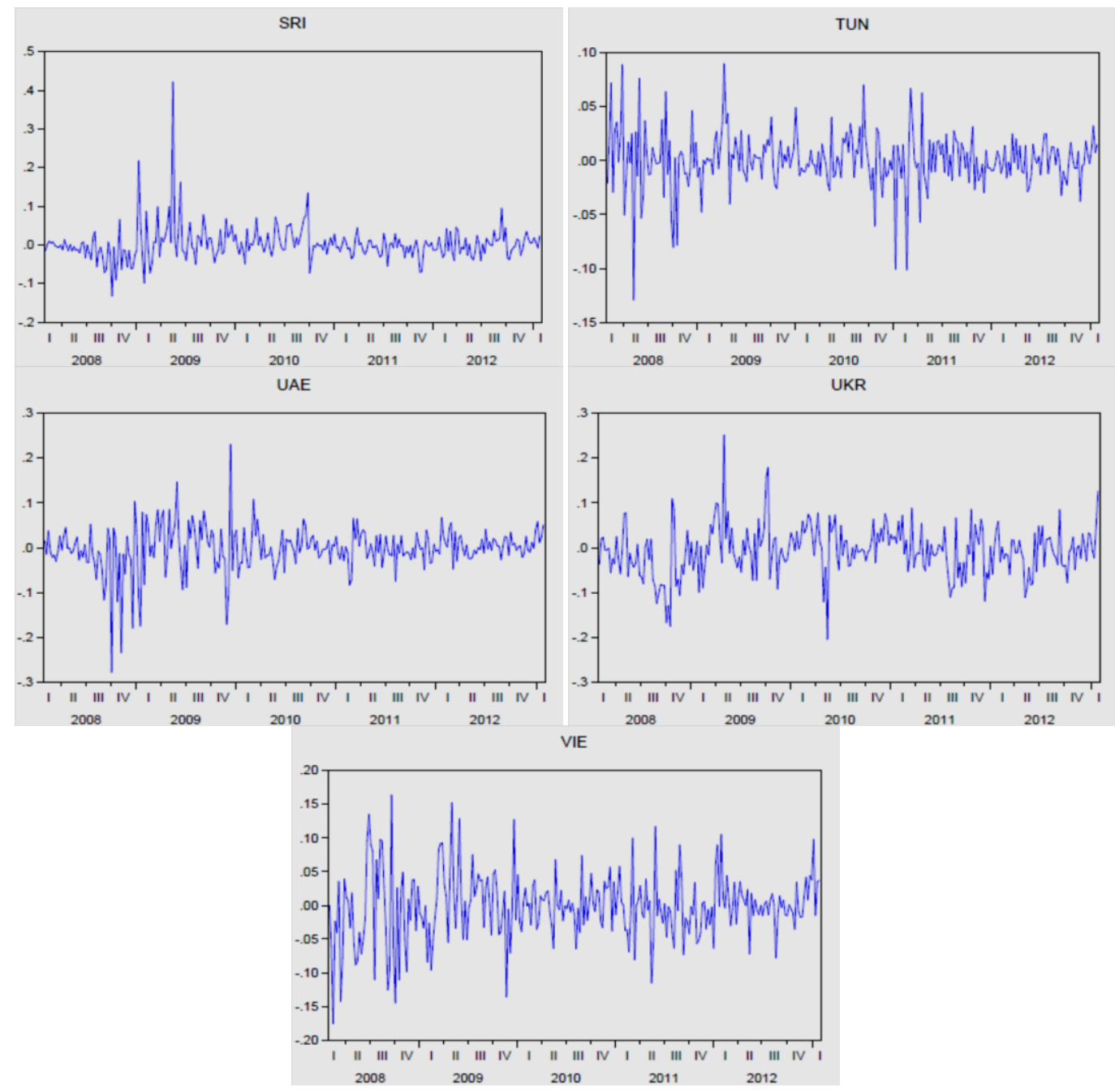


\section{NOTES}

